\title{
Incentive Mechanism Design for Distributed Autonomous Organizations Based on the Mutual Insurance Scenario
}

\author{
Yiguang Pan $(\mathbb{D})$ and Xiaomei Deng (iD \\ Department of Construction Management, Tsinghua University, Beijing, China \\ Correspondence should be addressed to Xiaomei Deng; dengxm@tsinghua.edu.cn
}

Received 29 March 2021; Revised 29 June 2021; Accepted 24 July 2021; Published 2 August 2021

Academic Editor: Dan Selişteanu

Copyright (c) 2021 Yiguang Pan and Xiaomei Deng. This is an open access article distributed under the Creative Commons Attribution License, which permits unrestricted use, distribution, and reproduction in any medium, provided the original work is properly cited.

\begin{abstract}
The rise of blockchain has led to discussions on new governance models and the cooperation of multiple participants. Due to the cognitive defects of the blockchain protocol in terms of intelligent contracts and decentralized autonomous organizations (DAOs), it is often unclear as to how to make decisions about the evolution of blockchain applications. Many autonomous organizations, with the support of network technologies such as blockchain, blindly absorb members and expand the scale of the capital pool, while ignoring the cost advantage of traditional autonomous organizations based on social relations and mutual supervision to fight information asymmetry. In this context, this study analyzes the evolutionary trend of autonomous organizations and their members' strategies under different policy environments. To this end, under the digital economy background, based on game theory, the evolutionary dynamics method, and the form of the mutual insurance organization, this study constructs an evolutionary dynamics model of distributed autonomous organizations. The results show that blind expansion without review aggravates the overall risk pool's moral hazard, in the context of mutual insurance. Organizational strategies, such as risk pool splits, can effectively improve the risk pool's operating performance and establish a benign competition elimination mechanism. Driven by cooperation efficiency and split supervision based on homogeneous clustering, the comprehensive application of the market elimination mechanism can effectively combat moral hazards, restrain the adverse effects of member flow, expand the living space of small- and medium-sized insurance organizations, curb the emergence of a large-scale monopoly risk pool, and improve market vitality. These conclusions and suggestions also apply to autonomous organizations based on social relations and mutual supervision. The results offer specific decision-making guidance and suggestions for the government, insurance companies, and risk management.
\end{abstract}

\section{Introduction}

Various mutual and peer-to-peer (P2P) insurance companies and other decentralized autonomous organizations (DAOs) or decentralized autonomous companies (DACs), typified by the use of blockchain, smart contracts, and other digital technologies, have been blindly absorbing funds and expanding while ignoring the participation of organizational members in autonomy. This reflects a severe departure from the objective of establishing an autonomous organization and its organization and operation. In addition, the recent large-scale withdrawal of the members of online mutual aid platforms and the flight of P2P financial organizations have demonstrated the need to supervise online mutual aid and similar platform autonomous economic organizations.

Few studies have analyzed the given context against the background of the digital economy by applying game theory or combining specific application scenarios. However, there has been a lack of emphasis on the specific organizational structure. The concept of distributed autonomous organizations has recently received attention with the rise of blockchain technology and various platform economies. Since its emergence, mutual insurance has exhibited the key elements and characteristics of a DAO, such as a one-person vote, openness and transparency, and a decentralized autonomy, under the background of the digital economy proposed at present. Thus, mutual insurance fits the context 
of the blockchain technology, DAO, and platform economy business model. However, an expansion of the capital scale weakens the relationship between the regions, kinfolk, and networks of the mutual insurance organizations based on traditional supervisory functions. These organizations have a high likelihood of facing serious moral hazards in the absence of adequate measures.

In this context, it must be noted that the DAO applications change the acceleration of organizational iterations, rather than completely abandoning the traditional corporate operation mode. For some time, the application scenarios of $\mathrm{DAO}$ are expected to maintain the existing legal and social structures of the organization and use the blockchain technology to complete transparency-related tasks such as record keeping. Given the significant time consumed to make adaptive changes through the legal system, contract specifications, and governance structure, among others, DAOs can be used to implement organizational changes owing to their compatibility with the traditional business model. Additionally, DAOs are expected to offset the negative effects of capital expansion while accelerating organizational changes.

Organizations are composed of highly uncertain people; this uncertainty also exists in the DAOs. Owing to the high degree of human uncertainty and the complex decisionmaking process, it is impossible to encode DAOs fully into a smart contract, under the current technological level [1]. Despite accelerating information dissemination and organizational iteration, DAOs are limited by the fact that most decision-making processes involving people may complete off-chain [2]. Particularly, a series of liability and risk issues (e.g., professional liability insurance) positively related to human uncertainty relies on constant interaction and repeated games between people to clarify their cognition.

In this context, as shown in Figure 1, we focus on the following problems that must be solved to promote the development of the digital economy: how to manage virtual organizations, establish a market mechanism of fair competition, maximize cooperation, and prevent monopoly operations? How does trust evolve in virtual organizations? How can we implement policy measures and realize their effects? In the next 5-10 years, DAOs may be able to solve these problems and code into smart contracts using blockchain technology [3]. This aspect also brings the subsequent quantitative analysis closer to practical application scenarios. The analysis focuses on how management ability contributes toward building trust and incentives. The mathematical framework captures the roles of management practices and the supervision system in team building, spinoff, bankruptcy, and other organizational measures. This study aimed to determine the key measures for successfully managing virtual organizations and their effect on the organization's evolution.

The remainder of this paper is organized as follows. Section 2 reviews the literature on the optimal organization size in relation to the governance of autonomous organizations. Section 3 constructs the evolutionary dynamics of autonomous mutual insurance organizations, based on game theory and an evolutionary dynamics model. Section 4 analyzes and discusses the model's results, and Section 5 provides conclusions and suggestions.

\section{Literature Review}

As shown in Figure 1, we begin with human community organizations, followed by mutual insurance organizations with special functional requirements, and subsequently move to distributed autonomous organizations under the block chain economy to review the existing literature. We selected papers for our review according to the keywords in Table 1. The papers were identified using the Web of Science, Wiley Online Library, and Microsoft Academic databases.

2.1. Threshold of the Average Size of Human Community Organizations. Transcending communities and races to facilitate large-scale cooperation supporting complete and efficient cooperation is the most significant human potential. Cooperation represents the underlying behavior support generated by monopolistic economic activities. Monopoly is caused by unregulated expansion of capital and ignoring moral hazards. Positive collaboration and cooperation serve as effective means and institutional guarantees to fight monopoly. In autonomous organizations, the optimal organization size is one of the critical issues for promoting cooperation. It is also one of the essential research questions in anthropology, sociology, and other disciplines. Researchers often use data on the neocortical volume, population size, and behavioral ecology variables to examine the relationships between the variables $[4,5]$. Dunbar [6] previously verified that group size is a relative cortical volume function, whereas ecological variables are not. In other words, the size of a community is not determined by the ecological environment in which a species is located, but it is more restricted by its cortical volume. Casari and Claudio [7] found that the upper limit of community size is 150 people, consistent with Dunbar's coefficient of 150 people. The social activities in human society still follow this rule, even with today's highly developed social network technology $[8,9]$. In online social groups, most personal networks present three or four circles as in the classical Dunbar structure, with a size comparable to the one initially described by Dunbar [20]. Small online autonomous communities with no more than 40 people can be managed democratically, but communities with more than 40 people need a leadership team structure [21].

\subsection{Upper Limit of the Average Pool Size of Mutual Insurance.} Similarly, the size of the primary risk pool of mutual insurance also has an upper limit. There is consensus on this finding in academia. However, the specific number and size depend on the research assumptions. In terms of experimental evidence, Abbink et al. [10] believed that the benefits of cooperation and mutual risk-sharing realized through an increase in scale can be offset by an increase in the number of free riders, thus creating a social dilemma. Murgai et al. [11], based on the analysis of water mutual insurance organizations in Pakistan, showed that community characteristics, 


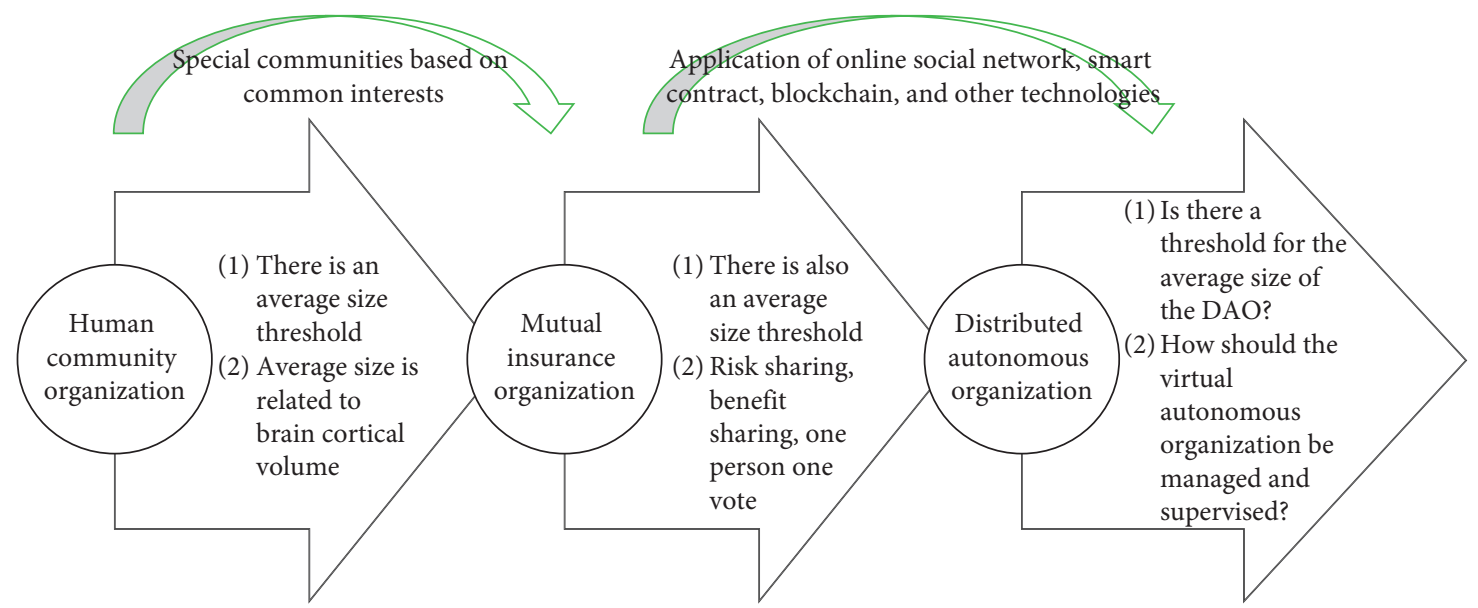

FIgURE 1: Existing research results and gaps.

TABLE 1: Related work on autonomous organization governance.

\begin{tabular}{|c|c|c|c|c|}
\hline Time & Keyword & Question & Comments & $\begin{array}{l}\text { List of } \\
\text { papers }\end{array}$ \\
\hline 1988-2021 & $\begin{array}{l}\text { Group size, cooperation, } \\
\text { social brain hypothesis, } \\
\text { common property resources, } \\
\text { transaction costs }\end{array}$ & $\begin{array}{c}\text { Is there an average size threshold for } \\
\text { autonomous organizations? } \\
\text { Does the development of social } \\
\text { networking technology affect the } \\
\text { threshold of the average size of } \\
\text { autonomous organizations? }\end{array}$ & $\begin{array}{c}\text { There is an average size threshold for } \\
\text { traditional autonomous organizations. } \\
\text { This threshold is related to brain } \\
\text { volume, but not to other ecological } \\
\text { variables. }\end{array}$ & {$[4-9]$} \\
\hline 1925-2017 & $\begin{array}{l}\text { Mutual insurance, group size, } \\
\text { cooperation, moral hazard, } \\
\text { information asymmetry }\end{array}$ & $\begin{array}{l}\text { Is there a threshold for the average risk } \\
\text { pool size of mutual insurance? }\end{array}$ & $\begin{array}{l}\text { There is also a threshold of the average } \\
\text { size for mutual insurance. This } \\
\text { threshold will vary by type of business, } \\
\text { but different types of mutual } \\
\text { insurance organizations have an upper } \\
\text { limit of average size. }\end{array}$ & [10-19] \\
\hline 2017-2021 & $\begin{array}{l}\text { Distributed autonomous } \\
\text { organization, blockchain, } \\
\text { governance }\end{array}$ & $\begin{array}{c}\text { In the environments of blockchains } \\
\text { and other network technologies, how } \\
\text { is it possible to govern autonomous } \\
\text { organizations similar to mutual } \\
\text { insurance? }\end{array}$ & $\begin{array}{l}\text { In the blockchain economy, the } \\
\text { governance and supervision of DAOs } \\
\text { are still in the exploration stage. }\end{array}$ & {$[1-3,20-23]$} \\
\hline
\end{tabular}

transaction and cooperation costs, and other factors restrict the risk pool's size of mutual insurance. Through an empirical study, Adams et al. [12] showed that a mutual-system company's size correlates positively with the claim premium proportion. In other words, the larger the company, the higher the proportion of policy claims.

In terms of the theoretical model, Barigozzia et al. [13] proved that if there are optional cooperative strategies in the insurance market, then cooperation between policyholders can internalize incentives and positively impact premiums. Regardless of the pool size, cooperative strategies are the optimal equilibrium with the highest efficiency. From the perspective of pool size, small-scale pools can more effectively promote cooperation. Von Bieberstein et al. [14] also believed that, in the case of all kinds of homogeneous professional risk, while individuals obtain benefits from the risk pool, the risk pool scale is restricted by free-riding behavior that cannot be expanded unlimitedly. Using the lowest average risk and reducing the effect of moral hazard on the risk pool's return, Eisenhauer [15] deduced the optimal size of the risk pool to be 200-400. Aase [16] used game theory and other research methods. This proves that maritime mutual insurance companies' development trends evolve toward a medium scale under the equilibrium state.

In terms of the historical induction, by combining the development history of mutual insurance in the Netherlands for several centuries, van Leeuwen [17] found that the excessive size of the early guilds hindered the development of mutual insurance. For 1940-1960, the disorderly expansion of the organization size also reduced its ability to fight against moral hazard. As per Valgren [18], during 1900-1930, the legal size and capital requirements for setting up an agricultural fire mutual insurance in the United States were as follows: number of incorporators 25 to 50, property exceeding \$50,000, and minimum membership size of 25 . Guinnane and Streb [19] showed that the expansion of the 
German Mutual Insurance for Miners (KV) contributed to its continued decline in social foundation. In this case, a larger KV organization does not have a better way to deal with information asymmetry. Measures such as hospitalization and on-site verification improve the foundation to a certain extent, but they weaken the trust relationship between KVs and mutual organizations.

\subsection{Distributed Autonomous Organization Governance in the} Context of Blockchain. Governance is not straightforward in blockchains and DAOs due to their networked nature. Governance represents the framework for decision rights, incentives, and accountabilities to encourage desirable behavior in the use of resources [22]. However, research on the governance of DAOs is still relatively sparse, especially for the specific functional requirement of distributed autonomous organizations. The existing research mainly identifies the shortcomings of DAO governance. However, there are no corresponding governance measures. At present, there exist three misconceptions about the applicability of blockchain technology to many DAOs in general and the sharing economy in particular (trust-free fallacy, disintermediation fallacy, and consumer will fallacy). Blockchain technology does not inherently solve all trust issues when it comes to real-world interactions between human beings: the trust-free fallacy [23]. In autonomous organizations, including mutual insurance, there is a nonlinear relationship between the optimal scale and the threshold range that limits the size of the autonomous group, which is a necessary condition for sustainable cooperation behavior. However, the specific threshold distribution differs owing to the various assumptions of future distributed autonomous organizations based on blockchain technology, which will also be restricted by the organization's size. It is therefore very important to study DAO governance.

\section{Research Methods}

The Social Brain Hypothesis suggests that there is a "natural" group size of around 150 for humans [24, 25]. Many studies verify that the relationships in a user's personal network follow Dunbar's property or the rule of 150, with a comparable size [20]. There is considerable evidence that groupings of this size are characteristic of personal social networks, both offline and online [7, 8, 26, 27]. Therefore, the basic hypothesis is that the risk pool has a threshold of 150 members. The subsequent analysis is based on this hypothesis.

This study focused on the antimonopoly and incentive mechanism design of distributed autonomous organizations as a social dilemma under uncertainty. Since different DAOs have different organizational visions, mechanism analysis cannot be carried out under a unified framework. Hence, the mutual insurance organization, which is widely used and has extended organizational practice history, was taken as the research object.

3.1. Basic Assumptions and Conceptual Models. We built a mathematical model by combining the current operation scenarios of DAOs (Mutual DAO System, Aragon, and TeamBrella) using the blockchain and other technologies and based on the traditional mutual insurance model. We considered the organizational and management impacts of the gradual upgradation of technology applications in the next 5-10 years. We combined the basic assumptions with the actual operating scenarios (https://mutualdao.org/cn/, https://aragon.org/, https://teambrella.com/\#). Based on the above basic models, we undertook the following processes:

(i) First, we selected uncertain subjective risks positively related to people, such as professional liability insurance.

(ii) Second, we assumed the availability of digital currency and the payment of digital cash by the team members. The wallet's private key stores only the respective client system, and there is no external transmission.

(iii) Third, we set the threshold value of pool members to 150 ; it must be noted that the large scale of the meeting weakens the effect of mutual supervision among members.

(iv) Fourth, the participation of insurance members was divided into two types, cooperation and betrayal, to proxy for the actual level of risk and the level of concealment of their truthful public disclosure.

(v) Fifth, after the risk pool reached the threshold, we divided the pool into two or more new pools to prevent bankruptcy because of the weakening of the mutual supervision function.

Under the mutual insurance application background, the distributed autonomous system mainly includes members, systems, and fund layers. Figure 2 shows the composition and the operating mechanism of the DAO system. It is the primary medium for members to communicate, vote, make decisions, and exchange values. The capital layer represents a virtual capital pool built on digital currencies and smart contracts.

As shown in Figure 3, based on the DAO model's mutual supervision with the help of blockchain technology, members decide through voting and realize the organization's decision-making power. Even their voting rights can be delegated to other familiar members, thereby establishing a chain of trust. The application of digital technology makes it easier for members to develop mutual trust and eliminate intermediary agents and centralized fund circulation. Each member's claim is settled through other members' premium payments, significantly reducing unfair prices and malicious behavior.

As shown in Figure 4, each venture capital pool is mainly involved in survival competition. As the main index, the survival probability was measured by the average cooperation rate of the pool members. Pool-level events mainly included spin-off (fission into more than two pools) and bankruptcy (extinction). The prisoner's dilemma was the primary game played among the pool members, and the events at the individual-member-level mainly included the insurance (entering the pool), transfer (interpool flow), and 


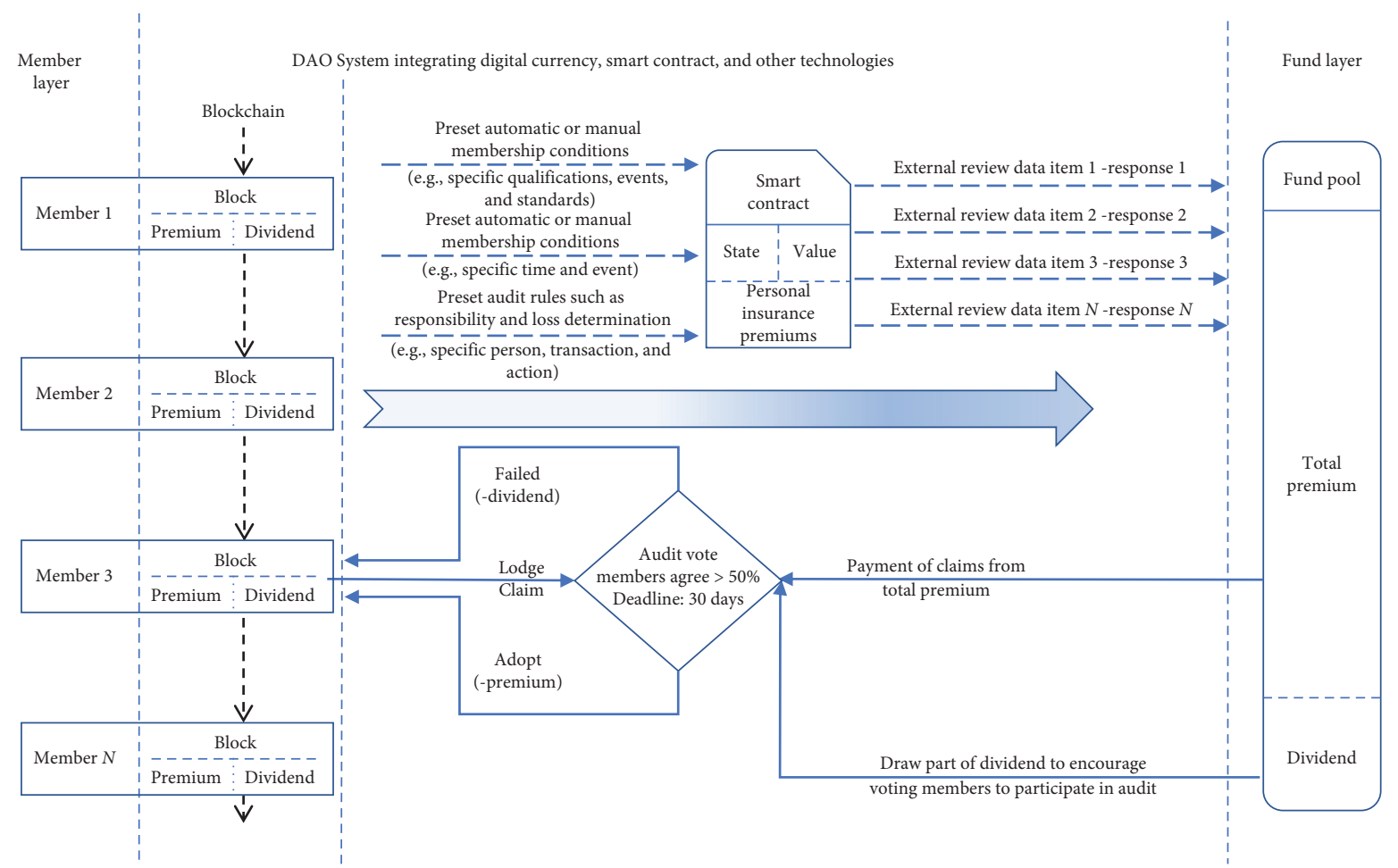

FIGURE 2: Mutual insurance application scenario based on a distributed autonomous organization. Note: in the blockchain economy, agreed-upon transactions would be enforced autonomously, following rules defined by smart contracts. The blockchain economy would manifest as a new form of organizational design, DAOs, which are organizations with governance rules specified in the blockchain. Member autonomy, one-person-one-vote rules, and the risk- and benefit-sharing principles of mutual insurance comprise an organizational form perfectly compatible with blockchain technology.

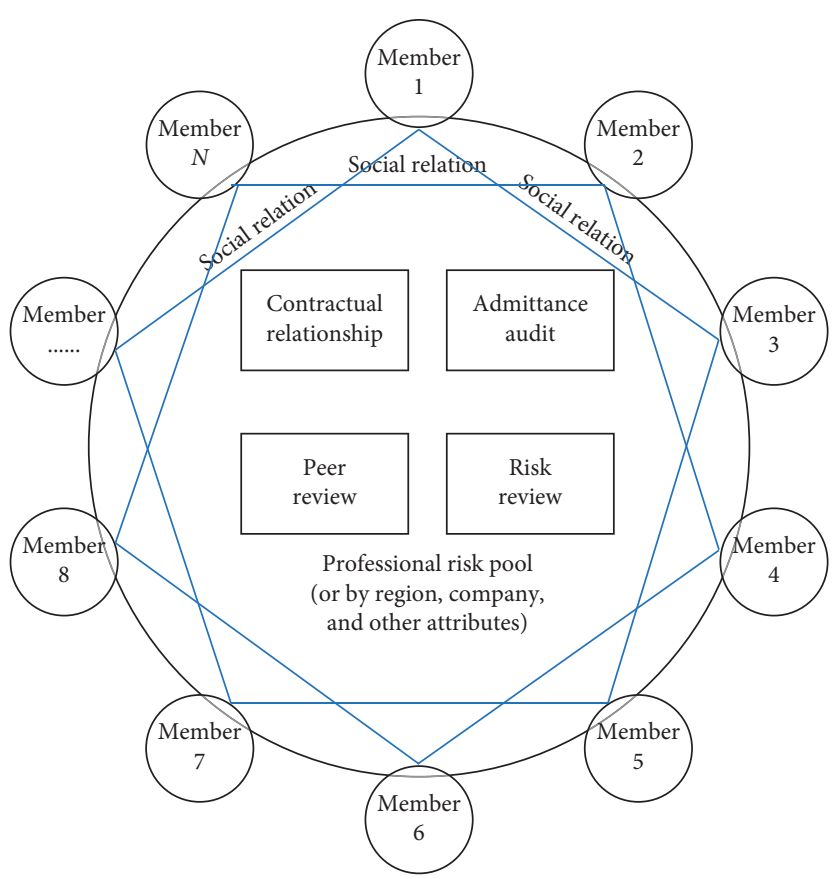

Figure 3: Mutual insurance autonomy model based on the mutual supervision function of social relations. Note: in addition to mutual insurance, autonomous organizations also have other forms, such as community economies and platform economies. 


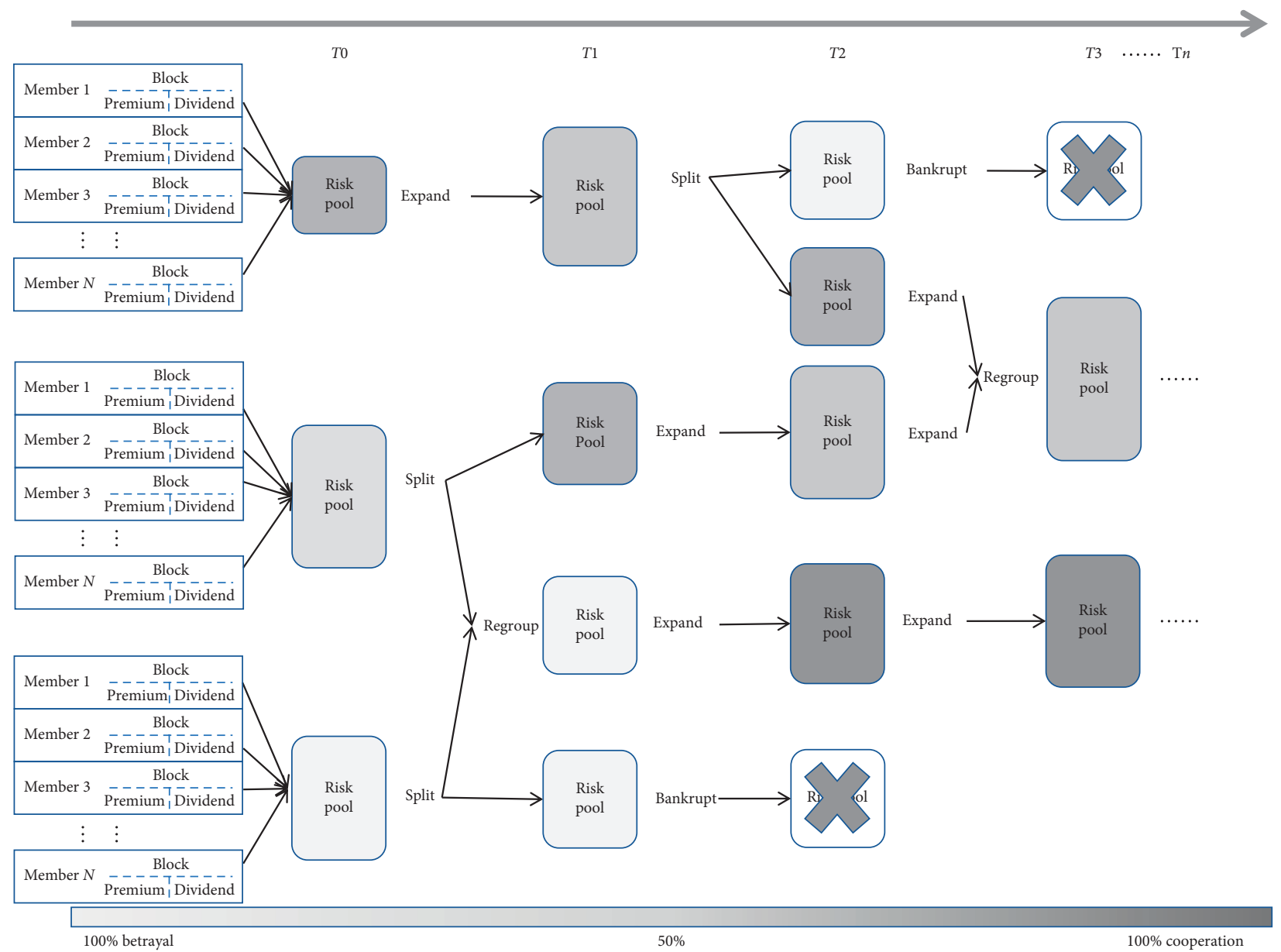

FIGURE 4: Interpool events and their conceptual model under the organizational strategy of the multilayer pool structure. Note: darker colors indicate a higher proportion of members' cooperation. The higher the proportion of members' cooperation, the higher survival probability of the corresponding risk pool.

elimination (getting out). We focused on the impact of the organizational and management measures taken after the essential risk pool size reaches the threshold (150 members) on the overall level of cooperation, trust, and pool capacity.

The color of the risk pool in Figure 4 represents the cooperation rate of its members in the pool. The higher the cooperation rate, the better the quality of the risk pool funds, the higher the probability of survival, and the higher the quality of participating members. Conversely, the lower the cooperation rate, the higher the proportion of the insurance fraud. The higher the probability of fraudulent insurance premiums, the worse is the corresponding risk pool funds' quality, and the greater is the probability of bankruptcy.

3.2. Mathematical Framework. Several scholars have integrated evolution with game theory to develop evolutionary game theory, a popular framework for describing individual interactions and overall changes in theoretical biology, economics, and sociology [28-39]. Based on the work of Damuth and Heisler [40], Okasha [41, 42], Champagnat et al. [43], and Simon [44], we constructed the two-layer mutual insurance pool selection and evolutionary dynamics models that adopt the classic prisoner's dilemma game at the individual and pool levels, respectively [43-47]. The research problem can be further abstracted as a prisoner's dilemma problem in taking members' premium payments as a public good to realize risk-sharing.

The model construction starts from the microdescription of the risk pool of the mutual insurance unit. Insured individuals' strategy characteristics directly affect their returns and indirectly affect the operational quality of the insured risk pool. The mathematical model, comprising the members of a mutual insurance risk pool (like a biological population), can be viewed as a population system with random interactions [48]. The state function $\theta_{t}$ describes the phenotype of each insured member. The insured individual and the overall phenotype of the risk pool at time $t$ depend only on the state function $\theta_{t-1}$ at the previous time. The interaction between individual members and the overall change in the risk pool can be observed by adjusting the Markov process parameters:

$$
\frac{\partial \theta_{t}}{\partial t}(x, y)=p_{t}(x, y)-\frac{\partial\left[\theta_{t} a_{t}^{c}\right]}{\partial x}(x, y)-\frac{\partial\left[\theta_{t} a_{t}^{d}\right]}{\partial y}(x, y) \text {. }
$$


In the differential equation of equation (1), $x$ and $y$ are real values and not integer values, respectively. They do not affect the overall modeling or the accuracy of the results. Simon [44] proved that the continuous deterministic model state is consistent with the results of the discrete random model. The pool can be understood as a unified organization with integer collaborators and traitors, close to its value $(x, y)$. In equation $(1), \theta_{t}(x, y)$ is the state function of the overall risk pool at time $t$. The right side of Equation $p_{t}(x, y)$ represents the events at the risk pool level, mainly including the $R_{(x, y)}$ establishment, spin-off, and bankruptcy of the risk pool. The establishment of the risk pool accompanies a larger pool split at the last moment, during which some risk pools may die out owing to a spin-off. The two partial derivative terms on the right side of the equation correspond to changes within the pool $R_{(x, y)}$, which is caused by the cooperative and defection strategies of members in the pool and the transfer of members between pools. In other words, the partial derivative terms correspond to the dynamics at the individual level, while the other terms correspond to the dynamics at the risk pool level:

$$
\left[\begin{array}{ll}
R & S \\
T & P
\end{array}\right]=\left[\begin{array}{cc}
b-c & -c \\
b & 0
\end{array}\right]
$$

The prisoner's dilemma game matrix among members in the pool is shown in equation (2), and the survival rate of members in the pool at time $t$ is proportional to their game payoff. $b>0$ denotes the cooperation benefits, and $c>0$ is the cost of providing public goods. If $c \approx 0$, there is almost no advantage in adopting a deception strategy, and if $c>b$, there is no prisoner's dilemma problem, given that we focus on the impact at the macropolicy level, and $b>c$ can simulate the dilemma of the real macrolevel public risk pool. The individual member and overall survival rate of the risk pool and indicators also depend on other parameters, and hence, the exact values of $b$ and $c$ are not so significant here:

$$
\begin{aligned}
& P(C \mid C)=r+(1-r) \frac{x}{x+y}, \\
& P(C \mid D)=(1-r) \frac{x}{x+y} .
\end{aligned}
$$

The game between members of an organization is not entirely random. It is related to the trust established on the basis of usual social relationships, reflecting the autonomy concept of mutual insurance based on social relationships. Individuals with high levels of trust are more likely to have interactive relationships. As shown in equation (3), the model uses the parameter $r \in[0,1]$ to characterize individuals' trust. It is described in mathematical language as follows: the probability $r$ that members choose the same strategy type and randomly select other members of different strategy types to conduct game interaction activities with a probability of $1-r$.

The expected returns of the cooperator and defector in the pool are as follows: $(x, y)$,

$$
\begin{aligned}
& \beta_{c}(x, y)=r(b-c)+(1-r)\left(\frac{x}{x+y}(b-c)+\frac{y}{x+y}(-c)\right) \\
& \beta_{d}(x, y)=r+(1-r)\left(\frac{x}{x+y}(1+b)+\frac{y}{x+y}\right) .
\end{aligned}
$$

The average survival rate of the cooperators and defectors in the pool $(x, y)$ is set to $s \beta_{c}(x, y)$ and $s \beta_{d}(x, y)$, and the parameter is the regulation parameter. $s>0$ here, where the survival rate not only represents the operation time of the pool but also determines its ability to absorb new members. The higher the survival rate, the longer the survival time, and the stronger the ability to absorb new members. The average dropout rates of both the collaborators and defectors were the same and proportional to the size of the group:

$$
\delta_{c}(x, y)=\delta_{d}(x, y)=d(x+y) .
$$

The model also includes the event in which members flow between different risk pools. The purpose of setting the turnover rate parameter is to simulate a malicious insurance fraud event that may exist in the insurance market through frequent flows to cover its high-risk nature. Each individual flows out of the original risk pool at a rate $\mu$ and randomly joins another risk pool. $C_{t}$ and $D_{t}$, respectively, represent the total number of cooperators and defectors in the population at moment $t$, and $P_{t}$ is the total number of members at moment $t$. The above values can be calculated using the state function $\theta_{t}(x, y)$ :

$$
\begin{aligned}
& P_{t}=\iint \theta_{t}(x, y) \mathrm{d} x \mathrm{~d} y, \\
& C_{t}=\iint x \theta_{t}(x, y) \mathrm{d} x \mathrm{~d} y, \\
& D_{t}=\iint y \theta_{t}(x, y) \mathrm{d} x \mathrm{~d} y .
\end{aligned}
$$

Correspondingly, the outgoing collaborator in any pool $(x, y)$ is $\mu x$, and the inflow is $\mu\left(C_{t} / P_{t}\right)$, where $C_{t} / P_{t}$ is the average number of collaborators included in the risk pool. Similarly, the betrayers flow out at a rate of $\mu y$ and flow into any risk pool at a rate of $\mu\left(D_{t} / P_{t}\right)$. The dynamic equation in the pool is expressed in the following equation:

$$
\begin{aligned}
{\left[\begin{array}{l}
x^{\prime} \\
y^{\prime}
\end{array}\right] } & =\left[\begin{array}{c}
\left(s \beta_{c}(x, y)-\delta_{c}(x, y)\right) x+\mu\left(\frac{C_{t}}{G_{t}}-x\right) \\
\left(s \beta_{d}(x, y)-\delta_{d}(x, y)\right) y+\mu\left(\frac{D_{t}}{G_{t}}-y\right)
\end{array}\right) \\
= & {\left[\begin{array}{c}
a_{t}^{c}(x, y) \\
a_{t}^{d}(x, y)
\end{array}\right] . }
\end{aligned}
$$

The rate of the pool $(x, y)$ splitting of the model is

$$
f(x, y)=\lambda(x+y) \text {. }
$$


Splitting density is

$$
\begin{aligned}
h((x, y),(u, v))= & (1-\psi) h_{1}((x, y),(u, v)) \\
& +\psi h_{2}((x, y),(u, v)) .
\end{aligned}
$$

where $\psi \in[0,1]$ is the split correlation coefficient. When $\psi=0$, there is no correlation split, and all splits allocate two types of members in a completely random manner. When $\psi=1$, the fission density is $h_{2}$. At this time, all the collaborators will be in one part, and all the defectors will be in another part, that is, $h((x, y),(u, v))=\delta_{((x, y)=(u, 0))}+\delta_{((x, y)=(0, v))}$.

In the insurance market, the small pool is more likely to become bankrupt because of the low fund reserves in the competitive environment. This factor also explains why the insurance is set to the minimum capital supervision. In this context, it must be noted that excessive free-riding in the risk pool, owing to the tragedy of the commons, will lead to moral hazard and ultimately bankruptcy. To simulate the above real supervision and operation situation and observe the impact of relevant policies on the overall risk pool's evolution trend, we set the following parameters: the exit rate functions $e(x, y, P)$ increase with an increase in the number of risk pools, decrease with an increase in the risk pool's size, and decrease with an increase in group cooperators. This is similar to the selective extinction model in biology:

$$
e(x, y, P)=\frac{e_{0} P}{(\phi x+y)^{2}} .
$$

where $e_{0} \geq 0$ is the initial exit rate parameter and $\phi$ is the pool exit intervention coefficient. If $\phi=1$, the exit of the risk pool depends only on the size of the risk pool and is unrelated to the proportion of cooperation in the risk pool. If $\phi>1$, then the most cooperative group is in favor, and if $\phi<1$, the most noncooperative risk pool is more survivable.

Finally, the initial conditions of all risk pools in the overall mutual insurance market follow a two-dimensional normal distribution with the center $\left(C_{0}, D_{0}\right)$ :

$$
\theta_{0}(x, y)=\frac{N_{0}}{2 \pi v_{0}} e^{\left(\left(x-\bar{C}_{0}\right)^{2}+\left(y-\bar{D}_{0}\right)^{2}\right) / 2 v_{0}}, \quad(x, y) \geq 0 .
$$

In the initial member $P_{0}=\iint \theta_{0}(x, y) \mathrm{d} y \mathrm{~d} x<N_{0}$, the initial value can be controlled by parameters $v_{0}$ :

$$
P_{t}(x, y)=\rho\left(-f(x, y)-e\left(x, y, P_{t}\right)+\int_{x}^{\infty} \int_{y}^{\infty} \theta_{t}(u, v) f(, v) h((x, y),(u, v)) \mathrm{d} v \mathrm{~d} u\right)
$$

The first two items in brackets in equation (12) are the spin-off and exit rates of (8) and (10), respectively, and the third item is the establishment rate of a new risk pool $(x, y)$ accompanied by the spin-off event of a larger risk pool at the last moment. To simplify the analysis, we set the parameter $\rho$ to measure together the incidence of events at all risk pool levels. The overall model is equivalent to solving equations (1) and (12) and $\alpha_{t}^{c}(x, y), \alpha_{t}^{c}(x, y)$ in equation (7).

Equation (1) has no closed solution, but we can obtain the equation's numerical solution using the Monte Carlo method. The key is to set an appropriate number of iterations, that is, the value $T$. To ensure that the parameter $\theta_{t}$ is set, the final convergence to a stable state is also critical.

3.3. Model Parameters and Standard Values. We modeled the fixed-parameter setting. The unit of time $T$ represents the working day. Considering the actual situation and the convenience of calculation, we assumed that the average working life of insured members was 32 years. The average annual working day was 250 days, and the $T$ value was $32 * 250=8000$. Other parameters $b, c, d, s, e_{0}$ are given in the model. The selection of each parameter value follows the following constraints: the risk pool's average survival time is 3-30 years, and the unit risk pool members are between and 15 and 150 [17]. The above restriction conditions are abstract boundary conditions based on the actual situation and historical data of mutual insurance. For parameter values that were inconvenient to obtain or quantify, we considered using heuristics to indirectly determine some parameter values under the restriction of boundary conditions. We ensured that it matched the actual situation to the greatest extent and conducted numerical experiments on critical parameters to avoid meaningless results.

Variable parameters $(r, \rho, \phi, \psi, \mu)$ are distributed uniformly in value according to their respective attributes, and the specific value ranges are listed in Table 2 . The parameter combinations are $4 * 6 * 2 * 2 * 6=576$ species. The initial trust $r \in\{0,0.15,0.25,0.3\}$ represents different initial trust levels, which can be understood as the degree of familiarity established based on social relationships. The relationship between trust and cooperation is not a simple linear relationship. This can be understood as the probability of the interaction between individuals using the same strategy. After each iteration, we found a difference in the average trust in the overall risk pool. The average trust is $R_{t}=P(C \mid C)-P(C \mid D)$. In the context of mutual insurance, it can also be understood as the degree of heterogeneity among members. Simon explained $r$ in detail from the perspective of evolutionary biology [49].

The occurrence intensity of interpool events $\rho \in\{0,0.5$, $1,1.5,2,3\}$ represents the occurrence intensity of interpool events from zero to strong. The pool withdrawal intervention parameter $\phi \in\{1,1.5\}$, where $\phi=1$, means that the exit of the risk pool depends only on the size of the risk pool and has nothing to do with the other parameters of the risk pool. This can be interpreted as a completely market-oriented operation, and the size of each capital pool determines its viability. 
TABle 2: Parameter definitions and values.

\begin{tabular}{lccc}
\hline Parameter & & Definition & Values \\
& $r$ & Average trust among pool members & $\{0,0.15,0.25,0.3\}$ \\
& $\rho$ & The intensity of interpool events & $\{0,0.5,1,1.5,2,3\}$ \\
$\{1,1.5\}$ & $\{0,1\}$ \\
Change parameters & $\phi$ & Pool exit intervention parameter & $\{, 0.0001,0.00025,0.0005,0.001,0.003\}$ \\
& $\psi$ & Pool split correlation coefficient & 576 \\
\hline Parameter & $\mu$ & Interpool member turnover rate & 0.00025 \\
combination & $\lambda$ & Pool splitting rate regulation coefficient & 0.4 \\
\hline & $b$ & Choose the benefits of an honest strategy & 0.1 \\
& $c$ & Cost of choosing an honest strategy & 0.0005 \\
Fixed parameters & $d$ & Member exit rate parameter & 0.025 \\
& $s$ & Adjusting the coefficient of correlation between the growth rate & 0.025 \\
& $e_{0}$ & and strategic returns & 500 \\
& $N_{0}$ & Pool selective & $(2) 13$ \\
& $\left(\bar{C}_{0}, \bar{D}_{0}\right)$ & Composition of the initial risk pool member & 5 \\
& $v_{0}$ & Variance in the distribution of initial risk pool member & 8000 \\
\hline
\end{tabular}

Note: the numerical simulation can take a greater number of iterations. However, the number of iterations' $T$ value is high: the variable parameter combination $4 * 6 * 2 * 2 * 6=576$ cases. Hence, the overall amount of calculation will exponentially and significantly weaken the feasibility of the numerical simulation and the calculation accuracy (under the premise of giving attention to two or more things). It can also include members' entire careers and must cover most of the small- and medium-sized enterprises' lifecycle. $T=8000$ workday $\approx 30$ 年. In each iteration, we focused on selecting five critical indicators of the overall risk pool to store the number of iterations, the number of members who adopted the honest (cooperation) strategy, the number of members who adopted the deceit (betrayal) strategy, the degree of trust among the members of the overall risk pool, and the number of risk pools $-T_{t} D_{t} R_{t} N_{t}$. Total computational storage of data: $8000 * 5 * 4 * 6 * 2 * 2 * 6=23040 * 10^{3}$ groups.

For $\phi=1.5$, the operation performance of the risk pool can be obtained through system regulation, and the operational status of each risk pool can be identified. The exit mechanism is beneficial for the group with more operators and a positive elimination mechanism.

For $\phi<1$, a high moral risk is favorable to the risk pool; that is, the risk pool achieves a strong survivability when a high proportion of individuals adopt the betrayal strategy. To maximize the use of computation, extreme cases of reverse elimination were not considered in this study.

The pool splitting intervention coefficient $\psi \in\{0,1\}$ at time $\psi=0$ indicates that there is no correlation splitting and that all splits are assigned to members of both types in a completely random manner. When $\psi=1$, the cooperative members and the betrayal members are separated, and new risk pools are established. The flow rate of pool members $\mu \in\{0,0.0001,0.00025,0.0005,0.001,0.003\}$. If $\mu=0.0001$, the probability of any member flowing out of the risk pool was 0.0001 . In a 50 -member risk pool, this means that one member of the risk pool flows out once every 200 working days and flows in once with the same probability.

Despite restrictions on the model's function and parameter selection, the optional range of each variable parameter value is still relatively extensive from a mathematical point of view. To test the influence of the details of parameters on the model verification result, we also conducted a sensitivity analysis for the essential functions and parameters. The results of the numerical experiment showed that the operating results were quite robust within the model and fixed-parameter configuration environment. The calculation results of the five critical variable parameter combinations converged to a stable state.

\section{Results and Discussion}

The results of the numerical calculation showed that the introduction of measures such as splitting and bankruptcy at the risk pool level contributed to the formation of an overall risk pool competition mechanism. This can significantly improve organizational vitality in many aspects and is consistent with common sense and the reality of the market economy. The calculation results of the parameter index have the following aspects:

4.1. Pool-Level Events Can Significantly Promote the Emergence of Overall Cooperation. We selected three datasets: the benchmark group data $\rho=0, r=\{0,0.15,0.25,0.3\}$; the $r$ value in the two groups of control data remains unchanged, $\rho=\{1,3\}$. The benchmark group data indicated that all members were in a risk pool and there was no split event. The corresponding control group data indicated that, after the risk pool members reached a certain size, a split event occurred and the risk pool died during this period. The results showed that events at the risk pool level, such as spinoffs, can significantly improve members' comprehensive cooperation. The level of cooperation here refers to the percentage of members who adopt cooperative (i.e., cooperation) strategies in the model to the total number of members. A high level means that the higher the level of cooperation in the overall risk pool, the lower is the moral hazard of the overall risk pool.

As shown in Figure 5, even in the absence of trust $(r=0)$, interpool events can significantly promote the overall level of cooperation in the risk pool and effectively suppress the 


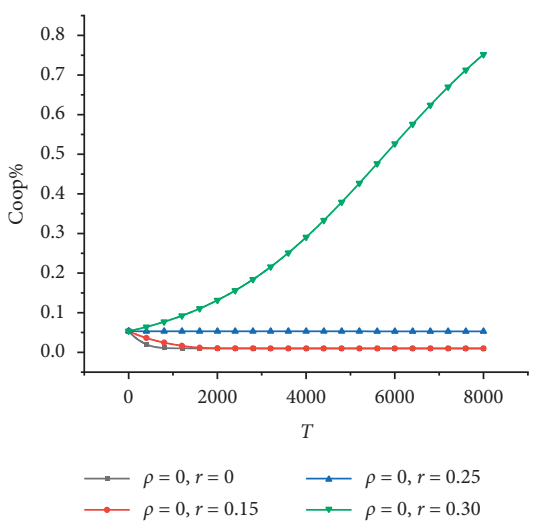

(a)

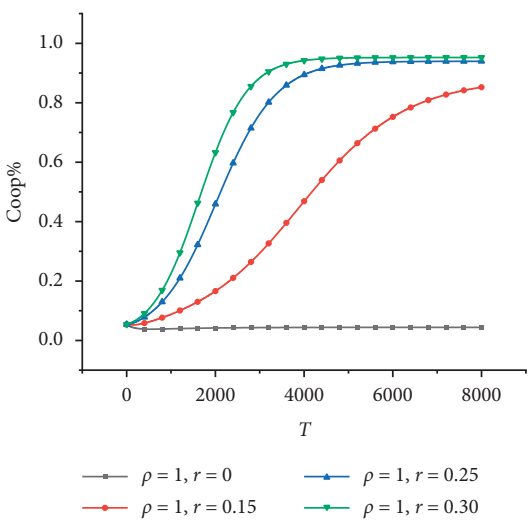

(b)

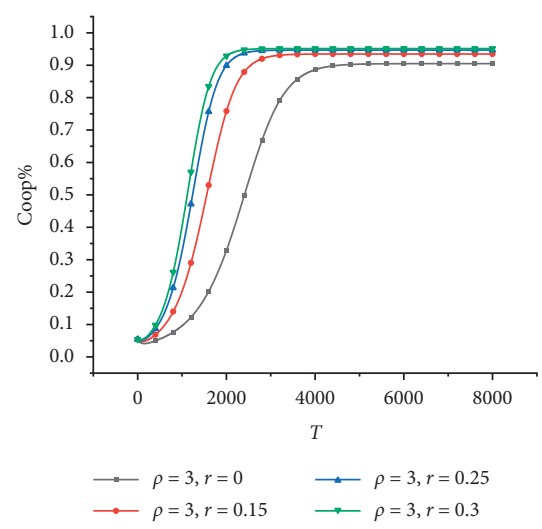

(c)

FIGURE 5: Influence of interpool event intensity on the overall cooperation efficiency. Note: different initial trust $r=\{0,0.15,0.25,0.3\}$, whether to take organizational measures ( $\rho=1$ means to take, $\rho=0$ means not to take) on the overall efficiency of risk pool cooperation. Pool-level events $(\rho=1)$ can significantly promote cooperation in the risk pool and effectively suppress moral hazard.

moral hazard. When there is no interpool event, cooperation will only occur when there is strong trust $(r=0.3)$. Consistently, the early mutual insurance was established based on strong relationships, such as kinship and geography. The early mutual insurance imposed restrictive regulations on the upper limits of the risk pool. The development of new technologies such as blockchain and social networks has only changed the way people communicate, speeding up the frequency of social interactions and the iterative update speed of events triggered by this development [50]. Therefore, the rise of blockchain and social networks has not substantially impacted the upper limit of the basic risk pool, and organizational measures must be taken to ensure that the overall risk pool's moral hazard remains low .

Frequent interpool events may be detrimental to the long-term development of the overall risk pool. Figure 6(c) shows that when $\rho=3$, the overall average pool capacity of the risk pool is significantly lower than that of the comparison group. Figure 6(b) shows that excessive interpool event intensity inhibits overall trust. Traditional organizational spin-offs, bankruptcies, and other events incur huge costs. The use of blockchain, digital currency, smart contracts, and other technologies significantly reduce the cost of organizational changes, efficiently facilitating the iterative upgradation of the organizational structure. At the same time, attention should also be paid to the frequent adverse impact of organizational-level events on organizational trust and scale expansion.

\subsection{Regulation of Pool Events Can Improve Multiple Index} Values of the Overall Risk Pool. Deregulation of the pool events means that the members of the two strategies are randomly assigned to more than two new risk pools when the pool splits. When bankruptcy occurs, the larger the scale, the stronger is the survivability. Figures 6(a)-6(c) show no regulation, while Figures 7 (a)-7(c) show the regulation of pool events. After the regulation, in terms of the level of cooperation, except when there are no interpool events (since the analysis background focused on whether the pool events regulate under the premise that there are interpool events, there is no interpool event $\rho=0$ only as a benchmark control). The adoption of regulations can significantly improve the convergence speed of cooperation level indicators. After 2000 iterations, even under the basis of weak trust, the overall risk pool cooperation level maintained a relatively high equilibrium state.

After regulation, the overall trust level reached its peak after 1500-2000 iterations; this was followed by a slow up and down process and a subsequent convergence to a higher-level range. The overall trust level without regulation is shown in Figure 6(b). In the interval [0.28, 0.32], after regulation (Figure $7(\mathrm{~b})$ ), the trust level is in the interval of $[0.52,0.60]$, which can increase the trust level by approximately 0.3 units. In the context of mutual insurance, pool trust can also be interpreted as the level of homogeneity among members. The higher the value, the higher the insured members' homogeneity and the more consistent the members' risk attributes. Correspondingly, it is in the determination of premiums and risk management. There are more significant cost advantages in other aspects.

The regulation of pool events can also significantly increase the overall risk pool's average pool capacity, thereby increasing its size. After regulation, the average number of risk pool members increases from the $[32,43]$ interval in Figure $6(\mathrm{c})$ to the $[57,60]$ interval in Figure $7(\mathrm{c})$. The average number of risk pool members increases by approximately 15 .

4.3. The Movement of Members among Risk Pools Is Not Conducive to the Control of Moral Hazard. As shown in Figures $8(\mathrm{a}), 8(\mathrm{c}), 8(\mathrm{e})$, and $8(\mathrm{~g})$, when there is an interpool event $(\rho=1)$, without regulation $(\phi=1, \psi=0)$ and the flow rate $\mu=0$, various parameter combinations can promote cooperative development. When the flow rate $\mu>0$, under different initial trust levels, as the flow rate $\mu$ increases, there will be a delay in the development of the overall cooperation strategy. When the pool event is regulated $(\phi=1.5, \psi=1)$, as shown in Figures $8(\mathrm{~b}), 8(\mathrm{~d}), 8(\mathrm{f})$, and $8(\mathrm{~h})$, the influence of 


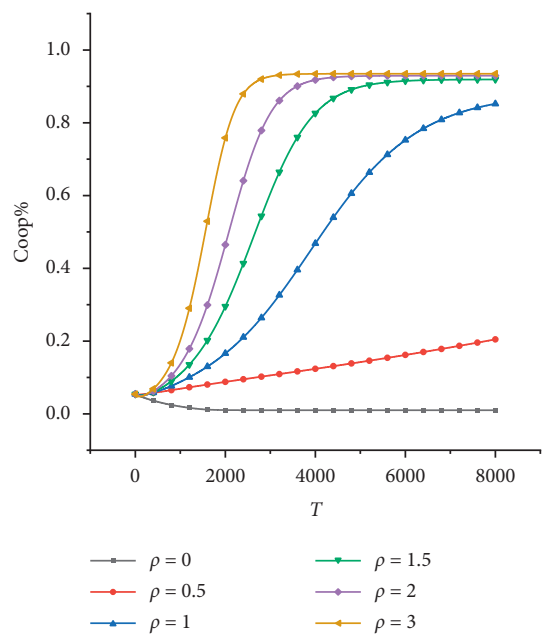

(a)

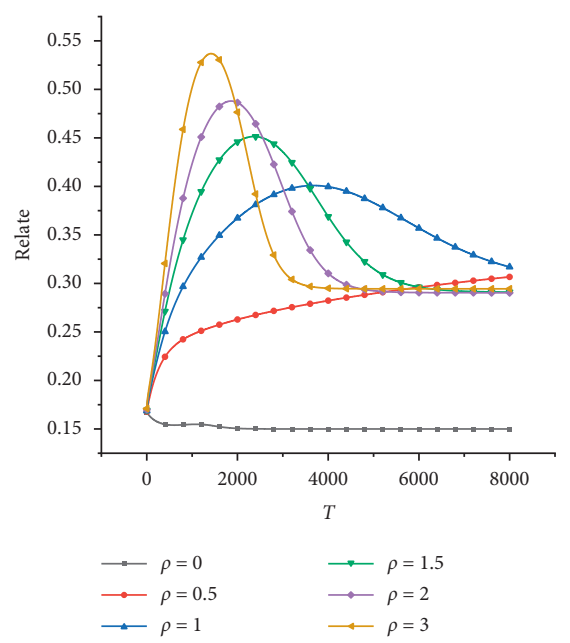

(b)
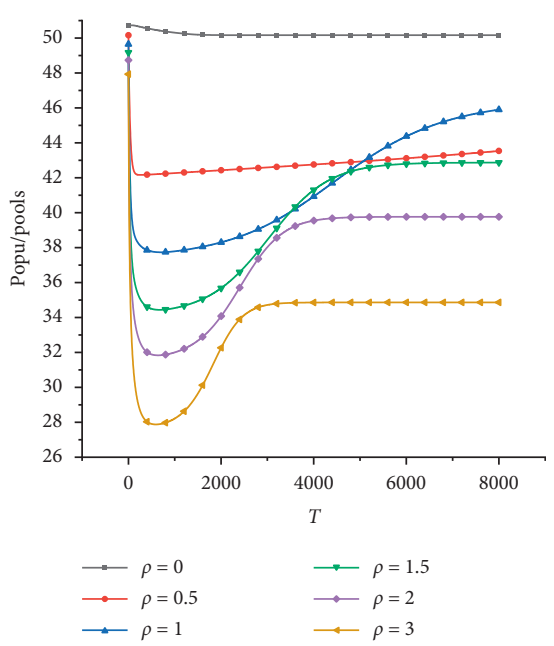

(c)

FiguRE 6: Influence of interpool events, such as spin-off, on overall cooperation efficiency, trust, and average pool capacity. Note: in the case of weak trust $(r=0.15)$, the influence of event intensity $(\rho=\{0,0.5,1,1.5,2,3\})$ on cooperation efficiency (coop\%, Figure $6(\mathrm{a}))$, trust (relate, Figure 6(b)), and average pool capacity (popu/pools Figure 6(c)) was analyzed. The results showed that pool-level events can improve the overall cooperation efficiency, trust level, and average pool capacity.

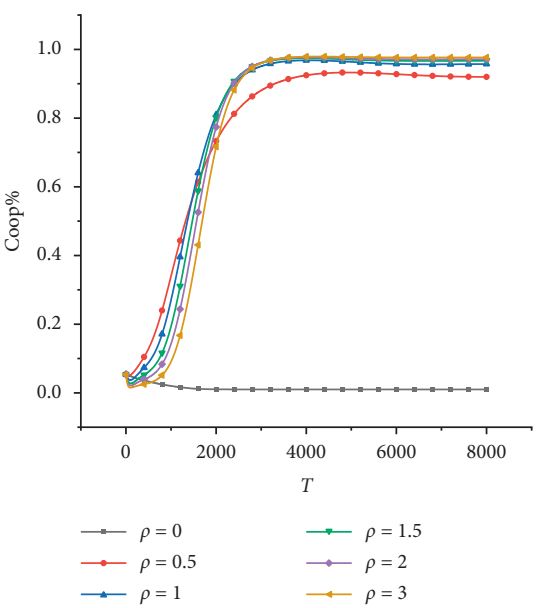

(a)

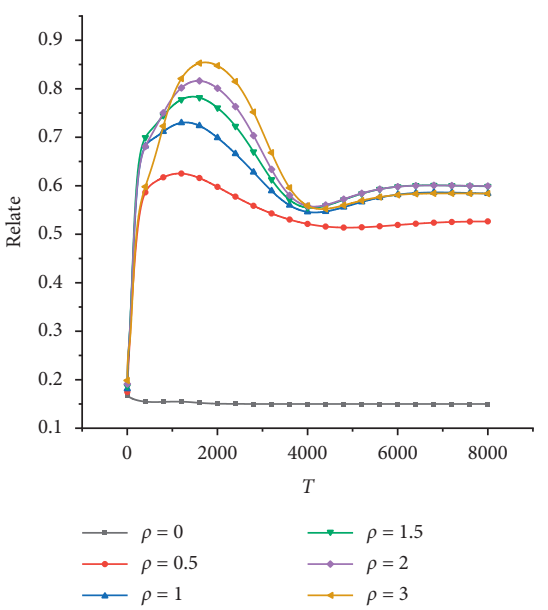

(b)

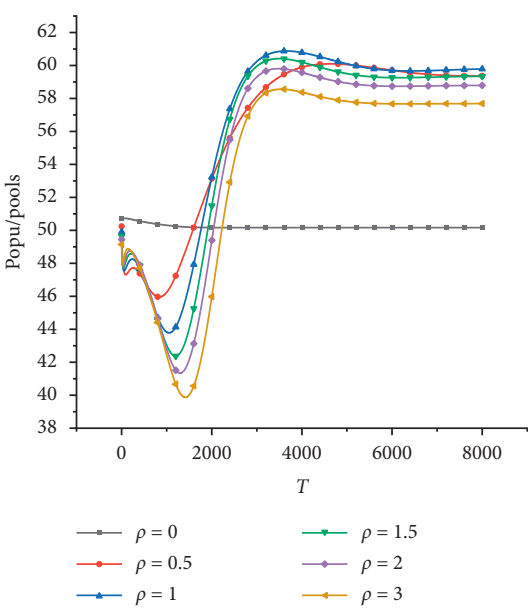

(c)

Figure 7: Changes in the overall cooperation efficiency, trust, and average pool capacity after the regulation of the pool exit and spin-off. Note: in the case of weak trust $(r=0.15)$, after adding the rules of pool exit and pool splitting (i.e., $\phi=1, \psi=0$ in Figure 6 , $\phi=$ $1.5, \psi=1$ in Figure 7), we analyzed the impact on the cooperation efficiency (coop\%, Figure 7(a)), trust (relate, Figure 7(b), and average pool capacity (popu/pools, Figure $7(\mathrm{c}))$. The results showed that the regulation of pool-level events $(\phi=1.5, \psi=1)$ can effectively improve the indicators of the overall risk pool.

the flow rate on the overall cooperation level becomes more complicated. The prohibition of interpool flow is not critical to the overall risk pool cooperation level. Even in the initial situation of trustlessness (Figure $8(\mathrm{a})$ ), a lower flow rate $(\mu=0.001)$ can also promote the overall cooperative behavior of the risk pool.

In the case of different initial trust values of $r$, the primary trend is as follows: a lower turnover rate will promote the formation of the overall risk pool cooperation strategy; beyond a certain threshold, it will hinder the development of cooperation. The specific threshold is not easy to quantify; even if a precise threshold is obtained, it cannot ensure a strong operability for the analysis of the macropolicy level. However, we can draw some basic laws of macroevolution of the turnover rate and cooperation strategy:

(i) The turnover rate parameter $\mu$ harms the overall cooperation development.

(ii) After regulating pool events, a low turnover rate can conditionally promote the overall risk pool cooperation strategy. 

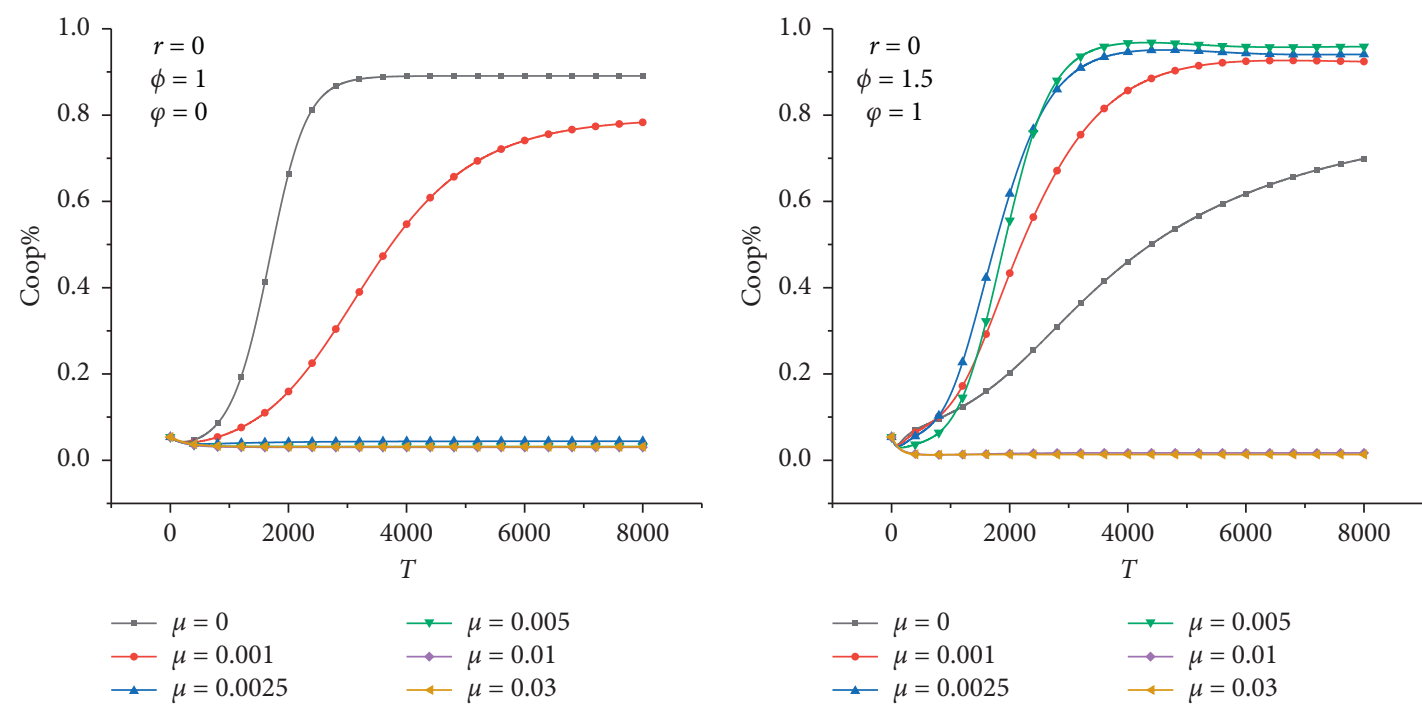

(a)

$$
\begin{array}{ll}
\longrightarrow \mu=0 & \rightarrow \mu=0.005 \\
\rightarrow \mu=0.001 & \rightarrow \mu=0.01 \\
\longrightarrow \mu=0.0025 & \multimap \mu=0.03
\end{array}
$$

(b)

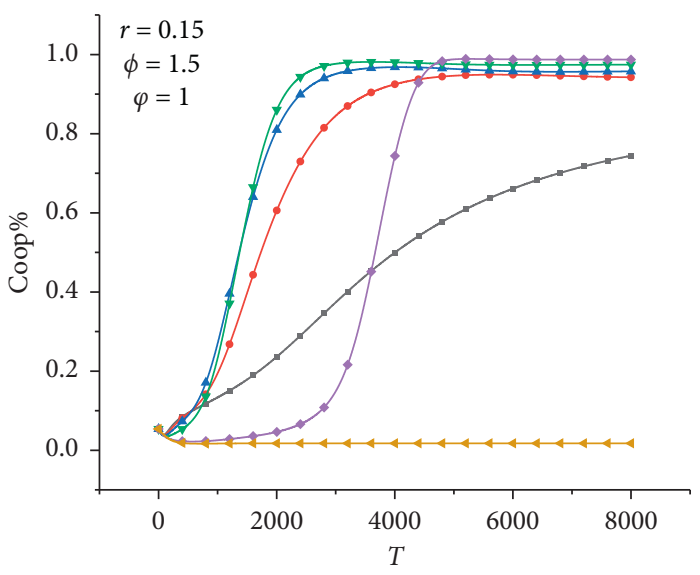

(d)

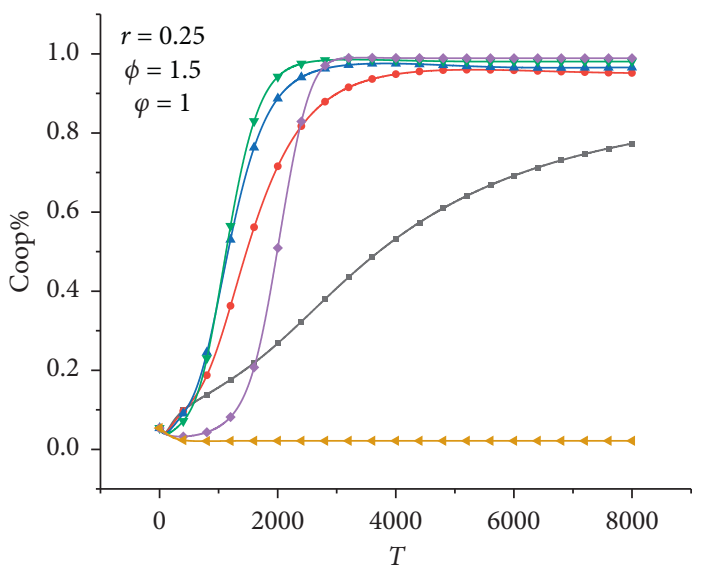

$$
\begin{array}{ll}
\rightarrow \mu=0 & \rightarrow \mu=0.005 \\
\rightarrow \mu=0.0001 & \rightarrow \mu=0.01 \\
\rightarrow \mu=0.0025 & \because \mu=0.03
\end{array}
$$

(f)

(e)

Figure 7: Continued. 


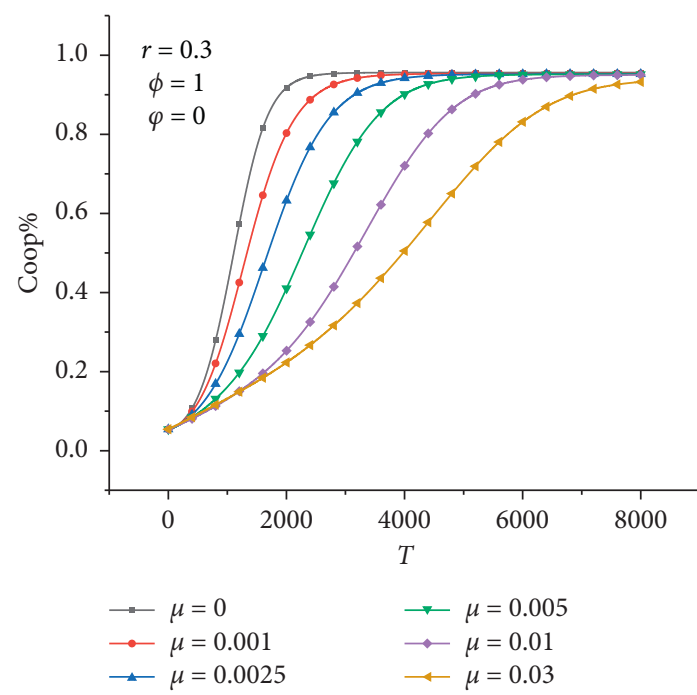

(g)

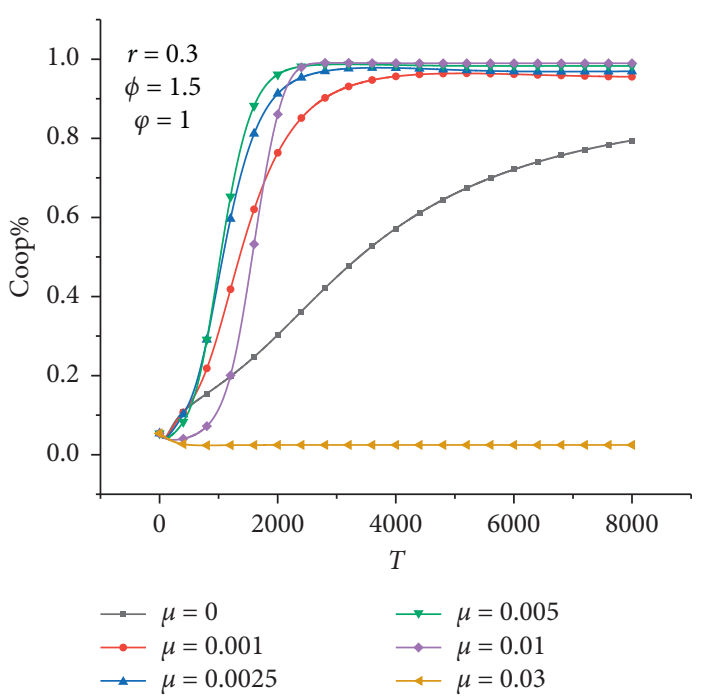

(h)

FiguRe 7: The influence of member flow on the overall cooperation efficiency before and after regulation under different initial trust levels. Note: initial distrust $(r=0)$, before and after regulation; the influence of member turnover rate $\mu$ on cooperation efficiency. Note: initial weak trust $(r=0.15)$, before and after regulation; the influence of member turnover rate $\mu$ on cooperation efficiency. Note: initial standard trust $(r=0.25)$, before and after regulation; the influence of member turnover rate $\mu$ on cooperation efficiency. Note: initial strong trust $(r=0.30)$, before and after regulation; the influence of member turnover rate $\mu$ on cooperation efficiency. Note: the calculation results under different parameter combinations showed that the turnover rate parameter $\mu$ had a negative effect on the overall development of cooperation.

(iii) The higher the initial trust, the higher is the threshold affecting the evolution of overall cooperation. In the initial stage of establishing the risk pool, the tolerance of the membership flow was relatively small. Conversely, in the middle and later stages of risk pool development, membership flow tolerance was relatively high after establishing overall trust.

Let us perform a simple logical thought experiment. Suppose there is no market elimination or member flow regulation in the real market, a free-market competition environment dominated by the capital scale. In such cases, there will be a huge risk pool. The mutual supervision function decreases with an increase in the scale. The freeriding strategy will demonstrate short-term profits, driving other honest members to change their original strategy. The free-riding strategy will invade a lot. If we allow it to develop freely for a long time, there will be a tragedy of the commons, which will eventually lead to the risk pool's rapid demise.

It must be noted that the regulation referred to in this study refers to the market elimination mechanism driven by the level of cooperation and the pool splitting supervision principle guided by homogeneous clustering. The aforementioned market mechanisms and supervision principles can effectively control the moral hazard of the overall risk pool. This coincides with the transition from "too large to fail" to "too centralized to fail" in various countries' current financial supervision. The elimination mechanism dominated by scale is one of the main factors that has helped the market to evolve and achieve a high degree of monopoly and engulf the people's interests (that is, "too big to fail"). The simulation results also prove that the market elimination mechanism can effectively control a moral hazard and expand the living space of small- and medium-sized insurance organizations to improve market vitality. This mechanism is oriented by cooperation level and is integrated with the supervision principle of pool splitting oriented by homogeneous clustering.

In terms of policy implementation, it is not feasible to prohibit members completely from flowing between risk pools, contrary to the organization and market principles of autonomy, sharing, and competition. To offset the adverse effects brought about by the free flow of members between the risk pools, the precondition is to establish a complete market competition elimination mechanism dominated by cooperation efficiency. At the same time, cooperation with the homogeneous clustering supervision measures of pool splitting, such as establishing a membership review system based on peer review before entering the pool, and appropriate restrictions regarding the flow of members in the early stage of the development of the risk pool will be lifted after trust in the middle and later stages develops to a higher level.

The Dunbar number is widely accepted in academic circles, and many scholars have verified its validity for online social network scenarios. The results of this study substantiate and extend the work of Guidi et al. [20] and Webber and Dunbar [21]. Webber and Dunbar found that in the social networking environment, autonomous organizations with professional collaborative needs cannot properly implement autonomous management for more than 40 people 
and must increase their management measures; however, the authors do not propose specific management measures. In line with this prior study, the present in-depth analysis of the specific mutual insurance scenario advances several specific governance measures, discussed in the following section.

\section{Conclusions and Recommendations}

The application of digital technology improves organizations' iterative update speed, but there is an upper limit on the social capacity of the human brain. This limitation restricts the basic units of the various DAOs based on social relations. After the social capacity exceeds the threshold value, organizational measures such as splitting and stratification must be taken to ensure that the number of members of the basic organizational units is below the threshold value. This will be crucial to ensure the overall cooperation efficiency of the organization and the effective exercise of mutual supervision and management function of members. It will also prevent market monopoly resulting from an excessive concentration of power. This conclusion is appropriate for most distributed autonomous organizations.

In the context of mutual insurance applications, this study draws the following conclusions:

(i) After the unit risk pool reaches the upper limit of the number of members, organizational strategies, such as splitting and stratification, can ensure the expansion of the overall risk pool and effectively combat moral hazard.

(ii) Even in situations where trust is weak, pooling events can lead to the emergence of overall cooperation.

(iii) If the intensity of the pool events is exceptionally large, it will inhibit the cooperation of the entire risk pool.

(iv) The regulation of pool events can simultaneously improve the cooperation level, overall trust, and average pool capacity of the overall risk pool.

(v) The interpool flow of members harms the construction of the overall risk pool cooperation strategy, but it is not a critical determinant.

(vi) At the same time, the market elimination mechanism dominated by cooperation efficiency and split supervision based on homogeneity clustering can effectively fight against and control the moral hazard, restrain the negative effect brought about by member flow, expand the living space of small- and medium-sized insurance organizations, restrain the emergence of the large-scale monopoly risk pool, and improve market vitality.

These findings have important implications for timely government regulation of virtual organizations through specific management measures and provide a theoretical roadmap for the future development of the digital economy.

Based on the abovementioned conclusions, we put forward the following specific recommendations from the perspective of distributed autonomous organization governance: (i) It is necessary to limit the number of risk pool members. After exceeding the threshold, it is necessary to implement management measures such as a spin-off and establish market mechanisms such as spin-offs and the elimination of the risk pool.

(ii) The intensity of risk pool splitting, mergers, and elimination should remain small enough to avoid vicious competition.

(iii) It is necessary to establish a market elimination mechanism with cooperation efficiency as the leading factor and decentralized supervision based on homogeneous clustering.

(iv) In the early stage of market development, it is necessary to appropriately control the flow of members among risk pools.

This study is also subject to certain limitations. For example, the model parameter setting was based on empirical values that may therefore have influenced our model estimates. This limitation can be addressed by future studies. Moreover, this study focused only on the mutual insurance scenario and offered governance measures, which may not apply to other autonomous organizations with special functional requirements. Future research may also explore the more universal governance of DAOs.

\section{Data Availability}

No data were used to support this study.

\section{Disclosure}

Any opinions, findings, and conclusions or recommendations expressed in this material are those of the authors and do not necessarily reflect the views of the sponsors.

\section{Conflicts of Interest}

The authors have no conflicts of interest to declare.

\section{Acknowledgments}

This material was based upon work supported in part by the National Natural Science Foundation of China under grants 71872094 and 71473145 .

\section{Supplementary Materials}

Part of the chart corresponding to the source code, file name "part1r0.m." (Supplementary Materials)

\section{References}

[1] L. Gratton, "Face the future of work," MIT Sloan Management Review, vol. 59, no. 4, pp. 96-95, 2018.

[2] L. Gratton, "Change Management," London Business School Review, vol. 28, 2017.

[3] MIT Sloan Management Review, What the Digital Future Holds: 20 Groundbreaking Essays on How Technology Is 
Reshaping the Practice of Management, MIT Sloan Management Review, Cambridge, MA, USA, 2018.

[4] R. Wade, "Village republics: economic conditions for collective action in South India." Geographical Journal, vol. 155, no. 1 , p. $120,1988$.

[5] J. Henrich, J. Ensminger, R. Mcelreath et al., "Markets, religion, community size, and the evolution of fairness and punishment," Science, vol. 327, no. 5972, pp. 1480-1484, 2010.

[6] M. D. Dunbar, "Neocortex size as a constraint on group size in primates," Journal of Human Evolution, vol. 22, no. 6, pp. 469-493, 1992.

[7] M. Casari and C. Tagliapietra, "Group size in social-ecological systems," Proceedings of the National Academy of Sciences of the United States of America, vol. 115, no. 11, pp. 2728-2733, 2018.

[8] G. Bruno, P. Nicola, and V. Alessandro, "Modeling users' activity on twitter networks: Validation of Dunbar's number," PLoS One, vol. 6, no. 8, Article ID e22656, 2011.

[9] D. Striga and V. Podobnik, "Benford's law and Dunbar's number: Does facebook have a power to change natural and anthropological laws?" IEEE Access, vol. 6, pp. 14629-14642, 2018.

[10] K. Abbink, B. Irlenbusch, and E. Renner, "Group size and social ties in microfinance institutions," Economic Inquiry, vol. 44, 2006.

[11] R. Murgai, P. Winters, E. Sadoulet et al., "Localized and incomplete mutual insurance," Journal of Development Economics, vol. 67, no. 2, pp. 245-274, 2000.

[12] M. Adams, L. F. Andersson, J. Y. Jia et al., "Mutuality as a control for information asymmetry: a historical analysis of the claims experience of mutual and stock fire insurance companies in Sweden, 1889 to 1939," Business History, vol. 53, no. 7, pp. 1074-1091, 2011.

[13] F. Barigozzia, D. Henriet, G. Pignataro et al., "Cooperation in risk-sharing agreements,” SSRN Electronic Journal, no. 765, 2011.

[14] F. Von Bieberstein, E. Feess, F. Fernando José et al., "Moral hazard, risk sharing, and the optimal pool size," Journal of Risk and Insurance, vol. 86, no. 2, pp. 297-313, 2017.

[15] J. G. Eisenhauer, "Risk pooling in the presence of moral hazard," Bulletin of Economic Research, vol. 56, no. 1, pp. 107-111, 2004.

[16] K. K. Aase, "Equilibrium in marine mutual insurance markets with convex operating costs," Journal of Risk and Insurance, vol. 74, no. 1, pp. 239-268, 2007.

[17] M. H. D. van Leeuwen, Mutual Insurance 1550-2015: From Guild Welfare and Friendly Societies to Contemporary Microinsurers, Palgrave Macmillan, London, UK, 2016.

[18] V. N. Valgren, "Farmers' mutual fire insurance companies," University Journal of Business, vol. 3, no. 2, 1925.

[19] T. W. Guinnane and J. Streb, "Moral hazard in a mutual health insurance system: German knappschaften, 1867-1914," The Journal of Economic History, vol. 71, no. 01, pp. 70-104, 2011.

[20] B. Guidi, A. Michienzi, L. Ricci et al., "Analysing dunbar circles in Facebook groups," in Proceedings of the 2021 IEEE 18th Annual Consumer Communications \& Networking Conference (CCNC), pp. 1-6, Las Vegas, NV, USA, January 2021.

[21] E. Webber and R. Dunbar, "The fractal structure of communities of practice: implications for business organization," PLoS One, vol. 15, no. 4, pp. 1-15, 2020.
[22] P. Weill, "Don't just lead, govern: how best performing organisations govern IT," MIS Quarterly Executive, vol. 3, no. 1, pp. 1-17, 2004.

[23] F. Hawlitschek, B. Notheisen, and T. Teubner, "A 2020 perspective on "The limits of trust-free systems: a literature review on blockchain technology and trust in the sharing economy," Electronic Commerce Research and Applications, vol. 40, Article ID 100935, 2020.

[24] R. I. M. Dunbar, "Coevolution of neocortical size, group size and language in humans," Behavioral and Brain Sciences, vol. 16, no. 4, pp. 681-694, 1993.

[25] R. I. M. Dunbar, “The social brain hypothesis," Evolutionary Anthropology, vol. 6, no. 5, pp. 178-190, 1998.

[26] R. A. Hill and R. I. M. Dunbar, "Social network size in humans," Human Nature, vol. 14, no. 1, pp. 53-72, 2003.

[27] R. I. M. Dunbar, V. Arnaboldi, M. Conti et al., "The structure of online social networks mirrors those in the offline world," Social Networks, vol. 43, pp. 39-47, 2015.

[28] M. J. Smith, "Group selection and kin selection," Nature, vol. 201, pp. 1145-1146, 1964.

[29] W. D. Hamilton, "Extraordinary sex ratios," Science, vol. 156, pp. 477-488, 1967.

[30] M. J. Smith and G. Price, "Logic of animal conflict," Nature, vol. 246 , pp. 15-18, 1973.

[31] J. Hofbauer and K. Sigmund, Evolutionary Games and Population Dynamics, Oxford University Press, Oxford, UK, 1998.

[32] J. Hofbauer and K. Sigmund, "Evolutionary game dynamics," Bulletin of the American Mathematical Society, vol. 40, pp. 479-519, 2003.

[33] M. A. Nowak and K. Sigmund, "Evolutionary dynamics of biological games," Science, vol. 303, pp. 793-799, 2004.

[34] S. Bowles and H. Gintis, "The evolution of strong reciprocity: cooperation in heterogeneous populations," Theoretical Population Biology, vol. 65, pp. 17-24, 2004.

[35] S. Bowles, "Group competition, reproductive leveling, and the evolution of human altruism," Science, vol. 314, pp. 15691572,2006

[36] X. Chen, A. Szolnoki, and M. Perc, "Probabilistic sharing solves the problem of costly punishment," New Journal of Physics, vol. 16, no. 8, Article ID 83016, 2014.

[37] X. Chen and M. Perc, "Optimal distribution of incentives for public cooperation in heterogeneous interaction environments," Frontiers in Behavioral Neuroscience, vol. 8, p. 248, 2014.

[38] X. Chen, A. Szolnoki, and M. Perc, "Competition and cooperation among different punishing strategies in the spatial public goods game," Physical Review E, vol. 92, no. 1, Article ID $12819,2015$.

[39] C. Elsenbroich and Nicholas Payette, "Choosing to cooperate: modelling public goods games with team reasoning," Journal of Choice Modelling, vol. 34, Article ID 100203, 2020.

[40] J. Damuth and I. L. Heisler, "Alternative formulations of multilevel selection," Biology and Philosophy, vol. 3, no. 4, pp. 407-430, 1988.

[41] S. Okasha, "The levels of selection debate: philosophical issues," Philosophy Compass, vol. 1, no. 1, pp. 74-85, 2006.

[42] S. Okasha, "Maynard smith on the levels of selection question," Biology and Philosophy, vol. 20, no. 5, pp. 989-1010, 2006.

[43] N. Champagnat, R. . Ferrière, and S. Méléard, "Unifying evolutionary dynamics: from individual stochastic processes to macroscopic models," Theoretical Population Biology, vol. 69, no. 3, pp. 297-321, 2006. 
[44] B. Simon, "A dynamical model of two-level selection," Evolutionary Ecology Research, vol. 12, no. 5, pp. 555-588, 2010.

[45] J. Damuth and I. L. Heisler, "Alternative formulations of multilevel selection," Biology and Philosophy, vol. 3, pp. 407-430, 1998.

[46] S. Okasha, Evolution and the Levels of Selection, Oxford University Press, Oxford, UK, 2006.

[47] B. Simon and M. Pilosov, "Group-level events are catalysts in the evolution of cooperation," Journal of Theoretical Biology, vol. 410, pp. 125-136, 2016.

[48] R. Durrett and S. Levin, "The importance of being discrete (and spatial)," Theoretical Population Biology, vol. 46, pp. 363-394, 1994.

[49] B. Simon, J. A. Fletcher, and M. Doebeli, "Hamilton's rule in multi-level selection models," Journal of Theoretical Biology, vol. 299, pp. 55-63, 2012.

[50] E. Vriens and E. Van Ingen, "Does the rise of the Internet bring erosion of strong ties? Analyses of social media use and changes in core discussion networks," New Media \& Society, vol. 20, no. 7, 2017. 